\title{
Reflets
}

Revue ontaroise d'intervention sociale et communautaire

\section{Le développement économique communautaire : nouveau départ pour le mouvement associatif Franco-Ontarien?}

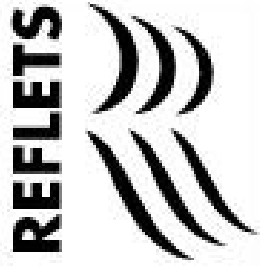

\section{Rachid Bagaoui et Donald A. Dennie}

Volume 5, numéro 1, printemps 1999

Pratiques et développement économique communautaire

URI : https://id.erudit.org/iderudit/026250ar

DOI : https://doi.org/10.7202/026250ar

Aller au sommaire du numéro

Éditeur(s)

Reflets : Revue ontaroise d'intervention sociale et communautaire

ISSN

1203-4576 (imprimé)

1712-8498 (numérique)

Découvrir la revue

Citer cet article

Bagaoui, R. \& Dennie, D. A. (1999). Le développement économique communautaire : nouveau départ pour le mouvement associatif

Franco-Ontarien? Reflets, 5(1), 75-94. https://doi.org/10.7202/026250ar

Tous droits réservés (C Reflets : Revue ontaroise d'intervention sociale et communautaire, 1999
Ce document est protégé par la loi sur le droit d'auteur. L'utilisation des services d'Érudit (y compris la reproduction) est assujettie à sa politique d'utilisation que vous pouvez consulter en ligne.

https://apropos.erudit.org/fr/usagers/politique-dutilisation/ 


\title{
Le développement économique communautaire: nouveau départ pour le mouvement associatif Franco-Ontarien?
}

\author{
Rachid Bagaoui et Donald A. Dennie \\ Professeurs, Département de sociologie et d'anthropologie, \\ Université Laurentienne
}

Les régions de l'Ontario connaissent aujourd'hui une double crise: celle, d'abord du modèle de développement (employeur unique, État et un modèle social et culturel) qui a caractérisé ces régions depuis la Seconde Guerre mondiale. Ce modèle est aujourd'hui en crise parce que l'État n'assume plus comme auparavant le développement et l'épanouissement de la population du Nord (peu de soutien financier aux organisations vouées au développement du Nord, coupures dans les services de santé et des services sociaux), ce qui entraine un manque de professionnels et de ressources de toutes sortes. De surcroît, le modèle de l'employeur unique ne peut plus assurer le développement de sa région comme auparavant (ex. croissance économique, création d'emplois, maintien des jeunes dans la région). Aujourd'hui, les usines n'embauchent plus massivement, rehaussent le niveau de qualification et réduisent leur force de travail. Par conséquent, le modèle social (et culturel) ne peut plus maintenir la cohésion sociale de la population: les jeunes quittent leur région faute d'emplois, la dépendance face à un employeur et aux politiques de l'État rencontre des obstacles, enfin, un manque probant de ressources humaines et financières touche notamment les services de la santé.

Le modèle de développement sur lequel se sont fiées les régions de l'Ontario est également en crise parce que les remèdes de la 
"...aujourd'hui, face au retrait de l'Etat et à la réduction des effectifs dans le secteur privé et les agences gouvernementales, la population des régions de l'Ontario est obligée de plus en plus de se fier à ses propres ressources et de créer sa propre économie.» part de l'État, des entreprises et des institutions financières appliquées à ces problèmes n'ont pas, malgré leur intentions louables, livré la marchandise promise. Bon nombre de ces remèdes se sont révélés à la longue coûteux, éphémères et surtout, impliquaient peu dans leur processus la population touchée par les problèmes. De plus, ces initiatives étaient axées sur des personnes cibles (comme de nombreux programmes de formation et d'aide sociale), laissant de côté de nombreux groupes sociaux. Pire encore, dans bien de situations, au lieu de permettre à la population de se prendre en main, ces mesures n'ont fait qu'aggraver la dépendance des gens affectés. De sorte qu'aujourd'hui, face au retrait de l'État et à la réduction des effectifs dans le secteur privé et les agences gouvernementales, la population des régions de l'Ontario est obligée de plus en plus de se fier à ses propres ressources et de créer sa propre économie.

La situation des régions de l'Ontario n'est pas unique. Dans d'autres provinces ou régions canadiennes comme au Québec ou en Acadie (Favreau et Lévesque 1996; Fontan 1994; Vaillancourt 1993; Greg 1996) ou ailleurs dans le monde comme en France et en Belgique (Laville 1992;Vienny 1994; Defouny 1994), la crise est une coordonnée de la réalité: effritement du travail de modèle fordiste, déclin de l'industrialisation modelée par les grandes entreprises, décroissance des services collectifs, retrait de l'État, crise de l'emploi, affaiblissement de la solidarité sociale. Ailleurs aussi, le développement économique communautaire (et l'économie sociale) apparait aujourd'hui selon plusieurs chercheurs comme une voie incontournable pour répondre aux difficultés des populations et pour redonner un nouvel élan à la solidarité sociale.

\section{Le développement économique communautaire}

Le développement économique communautaire (DÉC) differe des approches classiques (étatiques et privées) à de nombreux égards. C'est une stratégie qui désigne une approche basée d'abord sur la prise en main par une population de son propre 
"Plusieurs défenseurs de l'économie sociale vont même jusqu'à soutenir que ces nouvelles pratiques pourraient se révéler un modèle de développement innovateur de création d'emploi et de réponse à de nouvelles demandes sociales.» développement, puis sur la volonté de s'attaquer à toutes les formes possibles de marginalisation tant de la part des groupes concernés que dans les territoires touchés et enfin sur l'importance accordée aux aspects socio-économiques dans la stratégie de développement (Fontan 1994).

En dépit de leur grande diversité, ces initiatives de DÉC s'avèrent, selon plusieurs études, une composante essentielle dans la construction d'une nouvelle économie sociale, dans le sens qu'elles visent, contrairement au marché et à l'État, à intégrer des exigences économiques aux objectifs sociaux et ce, dans une démarche partenariale ainsi que locale et régionale. Plusieurs défenseurs de l'économie sociale vont même jusqu'à soutenir que ces nouvelles pratiques pourraient se révéler un modèle de développement innovateur de création d'emploi et de réponse à de nouvelles demandes sociales.

Si l'intérêt pour l'analyse du milieu économique communautaire au Québec et en Europe est notoire et se développe rapidement, il est toutefois peu marqué en Ontario où l'on en connait peu de choses, sinon que le milieu associatif est en train d'opérer dans son discours un renouveau vers l'économie et le partenariat (Cardinal et al.1994; Bagaoui 1997). Il n'existe pas, cependant, d'études systématiques qui brossent un profil global des organismes porteurs de ces initiatives ou d'études de cas suffisamment documentées.

Notre but est précisément de rendre compte de l'émergence de l'un des aspects de l'action communautaire en cours, à savoir le développement économique communautaire. Plus précisément, l'article veut décrire et analyser l'émergence, la crise et le renouveau de l'action communautaire. Compte tenu du manque de travaux sur le DÉC en Ontario français, notre démarche se veut exploratoire. Elle n'a d'autre ambition que de jeter les première bases à une problématisation du rôle du DÉC dans la construction d'une nouvelle économie sociale.

Les résultats que nous livrons ici s'appuient sur des recherches que nous menons depuis 1997 sur le développement communautaire. Nous avons réalisé pendant cette période quelques monographies d'organismes communautaires (voir les 
contributions de Lemieux, Dupont et Kihumbi dans ce numéro); un sondage auprès d'une vingtaine d'organismes de DÉC et un répertoire sur les initiatives de développement économique communautaire. Notre recherche s'appuie aussi sur des travaux historiques réalisés sur l'Ontario français depuis plusieurs années dans lesquels les historiens et historiennes ont tenté de reconstruire l'histoire des Ontariens et des Ontariennes de langue française depuis un siècle. ${ }^{1}$ Notre recherche s'est aussi appuyée sur des articles de journaux (principalement Le Voyageur et Le Droit).

\section{Les diverses générations ${ }^{2} \mathrm{~d}^{\prime}$ associations communautaires}

On peut distinguer, depuis le début des années soixante-dix, deux générations d'organismes communautaires qui s'inscrivent dans deux périodes historiques différentes. La première est née dans le sillage de l'animation socio-culturelle (à partir de 1969) et s'étend jusqu'à la fin des années 1980. La deuxième est apparue à partir des années 1990 (bien qu'ayant quelques antécédents dans la décennie précédente), dans un contexte de crise; il s'agit des organismes de développement économique communautaire (DÉC).

\section{Les associations communautaires axées sur une nouvelle dynamique entre l'État et la minorité canadienne-française}

On ne saurait aborder l'action communautaire sans la replacer dans la dynamique sociale qui s'instaure au cours des années 1960 entre l'État et la minorité canadienne-française. Si l'État accorde peu d'attention aux demandes sociales de cette minorité jusqu'aux années 1950, sa politique va bientôt changer à partir de la fin des années 1960 et devient à cet égard le foyer privilégié des revendications francophones. En effet, dès 1969 l'État fédéral assume la responsabilité d'établir et de gérer des programmes de langues officielles, d'établir les critères de financement, d'attribuer 
les subventions et d'appuyer le développement communautaire ${ }^{3}$. Quant au gouvernement provincial, il crée en 1967 une commission d'enquête sur la culture et les arts franco-ontariens qui se soldera par le rapport Saint-Denis qui recommandait entre autres l'établissement d'un Bureau des affaires franco-ontariennes au sein du Conseil des arts de l'Ontario (ce qui se réalisera en 1969). Quelques années plus tard, le gouvernement ontarien établira une deuxième commission d'enquête sur les arts qui produira le rapport Savard (1977).

La promotion du développement social et culturel des minorités de langue française par l'État canadien n'est évidemment pas le fruit du hasard; elle est d'abord conforme aux stratégies que poursuivait alors l'État dans la plupart des pays industrialisés, soit la prévention des crises par des mesures appropriées, la redistribution de la richesse et la promotion du développement social et culturel (Rosanavallon 1981). Ensuite, et c'est le point

"...cette promotion $d u$ développement social et culturel des minorités s'inscrit dans une période mouvementée de l'histoire du Canada où le pacte confédératif est remis...» sur lequel nous insistons, cette promotion du développement social et culturel des minorités s'inscrit dans une période mouvementée de l'histoire du Canada où le pacte confédératif est remis en question. En effet, pour répondre à cette crise de l'unité nationale, le gouvernement fédéral libéral avait établi l'une des commissions royales d'enquête les plus importantes de son histoire. La commission sur le biculturalisme et le bilinguisme, connue comme la commission Laurendeau-Dunton, identifie la crise linguistique qui sévit non pas comme un rapport entre une majorité et une minorité mais entre deux majorités. La commission souligne également l'écart entre les deux groupes linguistiques hors du Québec et l'importance d'accorder aux minorités linguistiques un traitement égalitaire. Le nouveau rapport de force entre les collectivités linguistiques aboutit à une reconnaissance des «deux peuples fondateurs» (Commission sur le biculturalisme et le bilinguisme) et d'une dualité linguistique juridique ${ }^{4}$.

Le développement du mouvement communautaire, au sens donné ci-dessus, s'inscrit dans ce nouveau rapport entre l'État et la minorité francophone qui s'est manifesté particulièrement entre 1969 et les années 1980. Bien que les associations de cette période s'inspirent de la même logique, à savoir le recours massif à l'État 
(État-nation et État-providence), elles n'ont pas évolué de la même manière.

Les associations des années 1970 étaient beaucoup plus un type de syndicalisme du cadre de vie. Les associations demandaient aux divers niveaux de gouvernement des équipements et services collectifs pour répondre aux besoins des communautés. Ces organismes communautaires sont symbolisés par les comités de citoyens qui ont pris naissance dans la région de Sudbury et qui se sont répandus un peu partout à travers la province. On les retrouve, par exemple, à Ottawa (le Comité du Réveil de la BasseVille, Le Coin du travailleur), à Vanier, à Rockland et à Toronto (le Comité Métropolitain de Parents et le Comité de parents SteMadeleine).

On ne saurait évidemment aborder le développement de ces comités sans les inscrire dans le projet de société véhiculé à l'époque et que l'on retrouve un peu partout en Amérique du

"Lancé en 1969 par le gouvernement fédéral, le programme d'animation se voulait une méthode d'intervention afin d'amener les minorités francophones à travers le pays à prendre conscience de leur situation socioéconomique et culturelle et de définir des actions pour la corriger le cas échéant.» Nord, soit celui de l'animation socio-culturelle. Lancé en 1969 par le gouvernement fédéral, le programme d'animation se voulait une méthode d'intervention afin d'amener les minorités francophones à travers le pays à prendre conscience de leur situation socio-économique et culturelle et de définir des actions pour la corriger le cas échéant. En Ontario, le programme d'animation profite largement à l'Association canadienne-française de l'Ontario (ACFO). Fondée en 1910 à Ottawa, sous le nom de l'ACFÉO, pour défendre des droits scolaires, cette dernière venait de changer son nom à l'ACFO (en 1968) dans le but d'élargir son action à d'autres domaines de la vie franco-ontarienne (Bureau 1989).

Dès ses débuts pourtant, l'animation socio-culturelle suscite de profonds déchirements au sein de la collectivité francoontarienne. À Sudbury, par exemple, les animateurs de l'ACFO régionale contribuent à l'établissement d'un comité de citoyens pour remplacer ou tout au moins redéfinir l'ACFO. Ce comité remettait ainsi en cause les élites traditionnelles qui avaient dirigé l'ACFO depuis ses débuts, son idéologie axée sur l'Église et l'école de même que ses méthodes d'action (Dennie 1972). Le comité propose de mettre l'accent sur la participation de toute la 
population : des jeunes, des ouvriers, des professeurs, des membres du clergé, des responsables de Caisses populaires, des professionnels, des assistés sociaux. Le Comité conteste également la logique centralisatrice imposée par l'ACFO provinciale, réclamant à sa place un regroupement de citoyens sensibles aux revendications et aux besoins spécifiques des gens du Nord de l'Ontario. Cette contestation, qui déborde la région de Sudbury, s'est exprimée pendant les années 1970 par des assemblées annuelles provinciales fort mouvementées.

Bien que l'idée n'était pas nouvelle, puisque des initiatives semblables existaient déjà au Québec (Favreau 1989), et bien que l'impact des comités n'était peut-être pas très significatif sur la vie de la population francophone de l'Ontario, leur portée sociale n'est pas à sous-estimer. Ces comités sont, en effet, révélateurs de la direction que prenait pendant cette période l'action communautaire: direction qui s'éloigne de plus des enjeux et des revendications des associations encadrées par l'élite traditionnelle. Les objectifs nationalistes, symbolisés dans la première vague des associations franco-ontariennes par les actions de l'Association canadienne-française d'éducation de l'Ontario fondée en 1910 pour défendre et promouvoir les droits scolaires des Canadiens français de l'Ontario (Choquette, Gervais 1993), qui prenaient la plupart du temps une coloration pan-canadienne, prennent des nouvelles dimensions dans la deuxième vague qui va des années

"Le concept identitaire change d'abord de Canadien français à Franco-Ontarien (ou Ontarois pour certains) et les objectifs s'élargissent à des champs nouveaux, hors de la seule logique de l'Église catholique et des écoles.» 1960 à la fin des années 1980. Le concept identitaire change d'abord de Canadien français à Franco-Ontarien (ou Ontarois pour certains) et les objectifs s'élargissent à des champs nouveaux, hors de la seule logique de l'Église catholique et des écoles. En effet, par leurs interventions dans l'amélioration des conditions de vie des citoyens et des services collectifs: services de transport, de média francophones, d'entretien domiciliaire, de services pour les aînés, de construction de centres communautaires, des chemins de fer, des réseaux d'onde, etc., ces comités tentent d'arracher les associations du paternalisme des élites et de l'encadrement paroissial. À Sudbury, par exemple, un comité d'action né dans le giron du Comité des citoyens revendique auprès de la Commission de la radio-télévision du Canada et obtient de la Société Radio- 
Canada un poste de radio pour desservir les francophones du nord-est de l'Ontario. Ce poste - CBON - diffuse depuis septembre 1978 à Sudbury.

Les années 1980 se caractérisent par le début d'une crise structurelle dans le domaine des politiques sociales et de l'engagement de l'État (Guest 1995).Au Canada et un peu partout dans les sociétés industrialisées les autorités publiques commencent sérieusement à remettre en question les fondements de la gestion des rapports sociaux, gestion héritée de l'après-guerre, et l'appui à la minorité ne va pas toujours de soi. Le gouvernement provincial adopte une politique d'étapisme dans l'établissement d'organismes dans les domaines judiciaire (la loi adoptée en 1984) et de la santé (aucun véritable progrès avant 1989), des communications (la Chaîne française de TVO entre en ondes en 1987). Malgré cela, les associations communautaires ne ressentent pas encore durement les effets de la crise.Au cours de ces années, en effet, elles survivent surtout sous le gouvernement libéral provincial qui accède au pouvoir en 1985 grâce à l'appui du Nouveau Parti démocratique, elles se diversifient et elles tendent de plus en plus à s'enraciner dans les nouvelles réalités franco-ontariennes (Juteau et SéguinKimpton 1993; Carrière 1993).

Bien que s'inspirant de la même philosophie que les associations des années 1970, c'est-à-dire du recours à l'État comme foyer privilégié de revendications, leur démarche est différente. On ne demande pas seulement à l'État de développer des services collectifs; les francophones tentent, par le biais de regroupements de citoyens et de professionnels, d'offrir des services alternatifs à ceux proposés par les pouvoirs publics. On peut affirmer que cette époque est marquée par le développement de garderies populaires (cf. Coopératives Caroussel et Brin d'Herbe à Ottawa, la Garderie coopérative Arc-en-Ciel à Embrun, La Garderie coopérative Boîte à Soleil à Welland, Petite école française coop à Brampton, Les Petits Anges de Penetanguishene, l'école maternelle coopérative à Russell). À Hanmer, dans la région de Sudbury, les francophones se sont donné une coopérative alimentaire, de 1975 à 1985, suite aux efforts de l'animatrice de l'ACFO régionale et de nombreux Franco-Ontariens du milieu. 
Plusieurs autres coopératives de consommation se sont établies au cours de cette période (Coopérative Embrun, Coopérative de Hearst, Coopérative Pointe-aux-Roches, Coopérative SaintEugène, entre autres). Des groupes de Franco-Ontariens de tous âges et de différents milieux fondent aussi des coopératives d'habitation dont la Place Verchères à Sudbury, L'Arbre de vie à Toronto, la Coopérative d'habitation Saint-Georges à Ottawa, la Coopérative Beauparlant à Welland, Terre-nouvelle à Curran; Lafontaine à Gloucester, DesJardins à Ottawa.

Le mouvement communautaire allait aussi se développer dans le domaine de l'alphabétisation. Le coup d'envoi était donné par une expérience communautaire née en 1982 à Hawkesbury. Un comité d'alphabétisation Prescott et Russell a établi et défini le problème de l'alphabétisme comme un problème socio-culturel d'abord, nécessitant une approche socio-éducative de type populaire. Un modèle alors expérimenté par les groupes populaires en alphabétisation du Québec a influencé ce projet. Le Comité d'alphabétisation prenait le nom d'Alpha-Action et allait organiser une action d'alphabétisation dans l'est de la province tout en posant le problème de l'analphabétisme des Franco-Ontariens au niveau des instances du milieu et de l'opinion publique. Ces initiatives seront consolidées et élargies en 1986 grâce au Plan d'action en alphabétisation. À la fin des années 1980, l'Ontario français comptait 19 centres d'alphabétisation.

Cette période est également marquée par l'apparition des radios communautaires. L'idée n'est pas nouvelle puisque dès 1973 surgissait à Hearst le projet d'une radio communautaire vite abandonné au profit d'un journal communautaire. Mais l'idée refait surface, au début des années 1980, à plusieurs endroits. Ce mouvement a été appuyé par la Fédération des Jeunes canadiensfrançais (FJCF) qui voyait la radio comme un moyen de lutter contre l'assimilation. L'Épinette Noire de Hearst et Radio Huronie de Penetanguishene constitueraient les premières initiatives dans cette direction. L'époque est aussi marquée par l'apparition des associations pour femmes. En 1989, la Fédération des femmes canadiennes-françaises de l'Ontario entreprend une vaste recherche-action afin de connaitre davantage la situation des 
"...lorsque la crise de l'État-providence s'amplifie au cours des années 1990, le mouvement communautaire francoontarien entre dans une phase d'incertitude et de tâtonnement.» femmes franco-ontariennes âgées de 45 à 64 ans. La Fédération, l'une des organisations traditionnelles de l'Ontario français (Brunet 1992), tente ainsi de redéfinir ses assises et son action communautaire (Garceau, Dennie, Tremblay, Charron 1991). Ce mouvement ne touche pas que les associations communautaires. Les professionnels, par exemple, se donnent aussi de nouvelles associations. Les enseignants étaient regroupés au sein de l'Association des enseignantes et des enseignants franco-ontariens depuis plusieurs années. Les avocats francophones, de plus en plus nombreux (Dennie 1972), établissent l'Association des juristes de langue française de l'Ontario en 1980. Les gens d'affaires établissement le Regroupement des gens d'affaires d'OttawaCarleton et deviennent aussi membres de la Chambre économique de l'Ontario.

Que retenir de cette trame historique? On doit constater que les associations se sont développées en fonction de contextes différents et pour tenter de doter les communautés francoontariennes d'une certaine complétude institutionnelle et organisationnelle. Leurs revendications se sont également diversifiées (logement, santé, éducation, etc.). Bien que leur apparition était brève, leur impact ne doit pas être sous-estimé. Enfin, ce qui caractérise cette période est que la plupart de ces organisations gravitaient autour de l'État. C'est de lui que dépendaient principalement leurs ressources financières et leurs orientations.Voilà pourquoi, lorsque la crise de l'État-providence s'amplifie au cours des années 1990, le mouvement communautaire franco-ontarien entre dans une phase d'incertitude et de tâtonnement.

\section{Crise et renouveau de l'action communautaire cours des années 1990}

Depuis le début des années 1990, la question de l'avenir des associations communautaires franco-ontariennes alimente de plus en plus le débat public en Ontario français. Et pour cause. Avec le problème de l'emploi, le retrait de l'État de plusieurs secteurs d'activités sociétales et la crise économique qui a sévi au début de la décennie, le milieu communautaire et associatif connaît des 
inquiétudes et des tâtonnements. Par conséquent, il est légitime de demander si les associations communautaires francoontariennes sont, dans ce contexte de crise, menacées ou si elles sont au contraire en train de se renouveler.

L'hypothèse soutenue dans ce texte, fondée sur certains indices, est qu'une véritable mutation est en voie de s'effectuer dans ce domaine et qu'elle pourrait ainsi mettre fin à une période de plus de 20 ans où le mouvement communautaire franco-ontarien s'est renouvelé et a élargi son champ d'action.

\section{L'action communautaires traditionnelle en crise}

«Bien qu'il soit difficile à ce stade-ci de mesurer avec précision ce phénomène, le milieu communautaire est d'avis que la situation financière s'est détériorée avec l'arrivée au pouvoir des Conservateurs à Queen's Park en 1995. Outre les problèmes financiers, la perte des acquis des services en français et les organismes francophones inquiètent profondément le milieu associatif.»
Les associations communautaires connaissent à l'heure actuelle un nombre important de problèmes dont le plus inquiétant est le financement ${ }^{5}$. Bien qu'il soit difficile à ce stade-ci de mesurer avec précision ce phénomène, le milieu communautaire est d'avis que la situation financière s'est détériorée avec l'arrivée au pouvoir des Conservateurs à Queen's Park en 1995. Outre les problèmes financiers, la perte des acquis des services en français et les organismes francophones inquiètent profondément le milieu associatif. Comme groupe minoritaire, la perte des acquis accentue, entre autres, les risques d'assimilation. En effet, les études sur le transfert linguistique sont nombreuses et démontrent en général que seules les régions limitrophes du Québec résistent tant bien que mal à l'assimilation (Juteau et Séguin-Kimpton 1993).

La situation financière oblige ces acteurs à s'investir davantage dans le travail. Ils sont contraints de consacrer chaque jour de nombreuses heures de travail, de faire du temps supplémentaire ainsi que s'occuper de toutes les tâches dans l'organisme. Et les bénévoles? Pas moyen de compter sur eux pour alléger leur fardeau car, disent les acteurs de ce milieu, le bénévolat semble en perte de vitesse. Les gens ne s'intéressent plus aux affaires francophones. Surtout les jeunes qui, selon plusieurs répondants et répondantes, savent à peine si ces organismes existent (Bagaoui 1997). 
Devant cette situation morose, la démobilisation semble le lot des acteurs du milieu associatif. Les répondants semblent «fatigués de se battre». Selon eux, «les choses ne bougent pas aussi rapidement qu'ils le voudraient»; ils se sentent seuls. Travailler dans un milieu minoritaire semble «une bataille sans fin». Plusieurs ont indiqué qu'ils «auraient dû faire un métier «normal». De plus, ils semblent ne plus savoir où «donner de la tête» sur le plan idéologique. Les problèmes quotidiens mobilisent davantage leur énergie. Ils n'ont plus le temps de s'impliquer dans les grands débats de société. Que pensent-ils, par exemple, du pluralisme culturel, de la mondialisation? Pas grand chose, sinon que ces problèmes ne les touchent pas. Les témoignages laissent donc entendre que c'est l'immédiat qui les mobilise (Bagaoui 1997).

Les conséquences créées par la crise de l'État-providence pèsent donc lourd sur l'action des associations communautaires. La situation est tellement morose que certaines d'entre elles, pour s'adapter au nouveau contexte, n'hésitent pas à adopter le langage des pouvoirs publics à savoir qu'il faut privatiser certaines activités culturelles pour concurrencer le secteur privé, «vendre» le bilinguisme des Franco-Ontariens pour attirer le financement, couper dans les dépenses, mettre l'accent sur les résultats et l'efficacité. On propose également de fusionner certains organismes, voire même de fermer ceux qui ne sont pas rentables. Il faut changer de priorités, de revendications pour répondre aux exigences des bailleurs de fonds. Afin de se faire entendre, les acteurs se donnent des instances provinciales, s'engagent dans le lobyying et participent à des consultations. ${ }^{6}$

En examinant les difficultés que vivent les acteurs dans le contexte actuel de crise, nous sommes tentés de conclure au déclin du milieu communautaire. Mais le déclin de quelles associations communautaires? Nous soutenons l'idée qu'il s'agit du déclin d'une génération particulière d'associations, celle des années 1970 et 1980, qui n'arrive pas à se défaire de sa culture étatique et qui continue de revendiquer que l'État demeure le foyer principal des revendications. Nous pensons, cependant, et certains indices le confirment, que d'autres associations communautaires tentent lentement aujourd'hui de refaire leur composantes sociales et 
d'inventer une nouvelle «culture» fondée sur la prise en main : il s'agit du développement économique communautaire.

\section{L'émergence du développement économique communautaire}

Le terme de

Développement

économique

communautaire

(DÉC) a fait son

entrée en Ontario

français au début des

années 1990.
Le terme de Développement économique communautaire (DÉC) a fait son entrée en Ontario français au début des années 1990. Il commence à apparaître dans certains débats ${ }^{7}$ et surtout à se matérialiser à partir de certaines pratiques socio-économiques comme les coopératives de travail, les entreprises communautaires de production et de services, les organismes de développement communautaire.

Les activités du DÉC sont nombreuses : initiatives d'appui et de création d'entreprises (la plupart du temps dans le domaine des services), centres culturels, construction de coopératives d'habitation, etc. (Cahier de ressources en DÉC 1996). Le DÉC existe sous diverses formes: coopératives, entreprises communautaires, organisation économique communautaire, etc. Certaines organisations comme les coopératives, et particulièrement les caisses populaires, remontent à très loin, mais la plupart sont nées dans les années 1980 et 1990, souvent en réaction à une crise comme la fermeture d'une usine, la pauvreté des femmes ou le chômage des jeunes.

L'initiative de créer l'organisme au départ vient dans la majorité des cas d'associations volontaires. Elles ont pour mission de créer des emplois et d'offrir des services aux entreprises. Parmi les activités ou services rendus à la population, mentionnons l'aide à la création d'entreprises, l'appui aux entreprises, les projets d'investissement, les programmes de formation, les projets de revitalisation de l'infrastructure communautaire et le renforcement des organismes communautaires. Les activités de ces organisations s'adressent à l'ensemble de la population du territoire desservi, régional et urbain. Ces organismes offrent des services à la 
"Le nombre total des bénévoles dans

l'ensemble des organisations étudiées dépasse une centaine de personnes. Quant au nombre d'employés, il dépasse aussi la centaine. La contribution des institutions financières à l'endroit de ces organisations est faible.» communauté dans sa langue. Le bénévolat constitue un élément important dans le fonctionnement de ces organisations. Le nombre total des bénévoles dans l'ensemble des organisations étudiées dépasse une centaine de personnes. Quant au nombre d'employés, il dépasse aussi la centaine. La contribution des institutions financières à l'endroit de ces organisations est faible.

Ce qui est visé par ces expériences, c'est le renouvellement des pratiques sociales allant dans le sens du développement d'un secteur communautaire comme un lieu de prise en main économique et d'affirmation culturelle. Plusieurs initiatives de développement communautaire existent. C'est le cas, par exemple, de Nordaski, une entreprise de développement économique communautaire qui a pris également naissance dans la nouvelle conjoncture (1988). Elle regroupe les communautés de Hearst, Hornepayne, Mattice-Val Côté et Constance Lake. Son objectif est de créer des emplois et de maintenir ceux existants. Pour ce faire, elle offre une gamme de services: formation de la maind'oeuvre, création d'une banque de données économiques, des renseignements sur les débouchés d'affaires, un centre de ressources et une banque de données générales. Le Nordaski soutient également la relance des entreprises locales; il peut accorder une aide financière allant jusqu'à $75000 \$$ et soutenir ces entreprises en leur fournissant l'information, la formation, l'aide administrative et technologique nécessaires à leur démarrage. Nordaski a des ententes avec les institutions financières (ex: Caisses populaires), la Banque fédérale de développement (BFD), le ministère de la Main-d'oeuvre et des ressources humaines. Nordaski est enraciné dans le milieu. Les plans de développement sont élaborés avec la participation des intervenantes et intervenants du milieu.

Cette stratégie de prise en main se traduit aussi, sur le plan local et régional, par l'existence des structures qui appuient le développement économique communautaire. À titre d'exemple, on peut mentionner le Conseil de la coopération de l'Ontario, qui réunit et chapeaute les organismes coopératifs franco-ontariens, qui a aussi procédé à une redéfinition de ses fonctions au cours des années 1990. Fort d'une subvention du gouvernement NPD, il essaie des nouvelles expériences, telles une recherche-action à 
«Si le DÉC semble une réalité nouvelle, les bases concrètes qui ont favorisé la reconnaissance de cette voie ne sont pas nouvelles; elles remontent aux années 1980.»
Sudbury pour reconstruire son conseil régional et territorialiser son mandat (Dennie 1997).

Si le DÉC semble une réalité nouvelle, les bases concrètes qui ont favorisé la reconnaissance de cette voie ne sont pas nouvelles; elles remontent aux années 1980.C'est le cas de La MaisonVerte, une compagnie de serres d'épinettes noires pour le reboisement, fondée en 1983 par un groupe de femmes actives dans l'association Parmi-elles, une association vouée à la création d'emplois pour les femmes de la région de Hearst. Le groupe de femmes fonda la compagnie avec l'appui financier du gouvernement fédéral (ministère des Richesses naturelles) et avec l'investissement de capitaux par des actionnaires locaux. Sa clientèle cible comprend les compagnies d'exploitation forestière. La compagnie emploie huit femmes à plein temps ainsi qu'une vingtaine de travailleurs saisonniers. La compagnie projette, en collaboration avec l'association Parmi-Elles, de mettre à la disposition des femmes de Hearst un fonds de création d'entreprises communautaires.

Du côté des coopératives, mentionnons Convergence, une coopérative de travail active depuis 1984 dans la région d'Ottawa. Elle se spécialise dans l'animation, la recherche, la formation, la planification stratégique, l'éducation et les communications. Sa transformation en coopérative remonte à 1991. Elle se veut non seulement un lieu pour favoriser l'accès au travail rémunéré pour les femmes par l'entremise d'une coopérative de francophones cogérée par les coopérants, mais aussi un lieu qui permet aux femmes de se prendre en main sur le plan économique.

Du côté des organismes communautaires, mentionnons l'expérience des Cuisines collectives de Hearst qui sont présentes depuis 1991. Plusieurs groupes expérimentent actuellement les cuisines collectives inspirées de l'initiative de HochelagaMaisonneuve à Montréal. Le projet s'adresse aux gens démunis (et non pas seulement aux chefs de familles monoparentales). À Hearst, le projet a vu le jour grâce à l'implication de tous les milieux; l'Église catholique, l'Église unie, l'Église anglicane, l'Église pentecôtiste et d'autres assemblées chrétiennes ont été rejointes. À cela s'ajoutent l'Association des locataires à prix modiques, la Société de l'aide à l'enfance, le Bureau du bien-être 
"Ces entreprises communautaires sont marquées non seulement par la diversité de leurs activités (insertion des jeunes, entraide, développement économique communautaire) et de leur statut juridique (entreprises communautaires, coopératives, organismes communautaires), mais aussi par l'importance accordée à la dimension économique.» social, le Centre d'embauche des jeunes, le Centre d'alphabétisation et le Rendez-vous familial. À la suite de nombreuses soirées d'information, un groupe représentant tous ces intervenants et intervenantes s'est formé pour la mise en marche du projet. Les membres (4 et 6 personnes) déterminent les recettes et cuisinent les repas. Cette initiative donne l'occasion aux membres de sortir de leur isolement, d'apprendre à cuisiner et surtout de faire des économies (Bagaoui 1997).

On peut évidemment multiplier les exemples (Bagaoui et Dennie 1998; voir également les monographies, réalisées sous notre direction, présentées dans ce numéro). Ce qu'il faut retenir, cependant, c'est que ces cas ne sont pas des réalités isolées, sans retombée sociale. Ils s'inscrivent dans le renouveau du discours et de l'action sur les orientations que prennent les associations communautaires franco-ontariennes. Ces entreprises communautaires sont marquées non seulement par la diversité de leurs activités (insertion des jeunes, entraide, développement économique communautaire) et de leur statut juridique (entreprises communautaires, coopératives, organismes communautaires), mais aussi par l'importance accordée à la dimension économique. Mais ce discours sur l'économie n'a rien à voir avec celui sur l'entrepreneurship apparu au cours des années 1980. En effet, on veut plutôt s'orienter vers la création d'emplois, la formation, la production des biens et services tout en continuant à répondre aux préoccupations sociales de la population francophone. Ce qui ressort également de ces expériences, c'est la prise de conscience par le milieu communautaire de la nécessité de sortir du cercle vicieux de la dépendance envers l'État et de chercher de nouvelles stratégies qui lui permettraient de s'approprier son propre développement. En ce sens l'action et le discours du milieu associatif semblent s'éloigner du discours des années 1970. Ce qui le caractérise, c'est son ouverture à de nouveaux thèmes comme le partenariat avec l'État et avec la majorité, la prise en charge et le développement socio-économique, sans pour autant renoncer à la question identitaire et les objectifs sociaux qu'ont toujours poursuivis les organismes franco-ontariens (Farmer 1996). Si les initiatives du DÉC peuvent être un levier incontournable 
pour répondre à de nouveaux besoins en français et fonctionnent dans une culture francophone, rien pour l'instant n'indique qu'elles sont en train de se révéler un nouveau modèle de développement comme le prétendent des études sur l'économie sociale. De sérieuses questions demeurent sans réponse et auxquelles il faut s'attaquer : ces pratiques peuvent-elles se généraliser à l'ensemble des associations communautaires? Comment se traduisent-elles dans la réalité? Plus important encore: ces initiatives signifientelles un véritable renouveau ou un simple palliatif à la crise de l'État-providence? Trouveront-elles de l'appui de la part des pouvoirs publics et du milieu? Quelles sont les retombées concrètes de ces initiatives? L'absence d'une voie alternative cohérente et efficace, susceptible de faire face au triomphe du néolibéralisme, ainsi que les difficultés que vivent les organismes de DÉC, incitent à la prudence (Boivin et Fortier 1998).

\section{Bibliographie}

BAGAOUI, R. et D. DENNIE (1998). Répertoire des organismes de développement économique communautaire dans le Nord-Est de l'Ontario, Première-Édition 1998, Sudbury, Université Laurentienne.

BAGAOUI, R. (1997). «Réponses des associations communautaires de la région de Sudbury à la crise de l'État-Providence», Reflets, vol. 3, no 1.

BÉLANGER P. R, et B. LÉVESQUE (1992). «Le mouvement populaire et communautaire: de la revendication au partenariat», G. Daigle et G. Rocher (s. la dir. de) Le Québec en jeu. Comprendre les grands défis, Montréal, Presses de l’Université de Montréal, 713-747.

BRETON, R. (1984). «Les institutions et les réseaux d'organisations des communautés ethnoculturelles», État de la recherche sur les communautés francophones hors Québec, Actes du premier colloque national des chercheurs, Fédération des francophones hors Québec, 4-20.

BRUNET, M. (1992). Canadiens-Français ou Canadiens Francophones? Une analyse des journaux de langue française de Sudbury, 1960-1975, Thèse de maitrise, Département d'Histoire, Université Laurentienne.

BUREAU,B. (1989). Mêlez-vous de vos affaires: 20 ans de luttes fanco-ontariennes, Association canadiennefrançaise,Vanier.

CARDINAL, L. et al. (1994). L'épanouissement des communautés de langue officielle, Ottawa, Ministère du Patrimoine canadien/Commissariat aux langues officielles.

CARRIÈRE, F. (1993). «La métamorphose de la communauté franco-ontarienne», Cornelius J. Jaenen (s. la dir. de) Les Franco-ontariens, 1960-1985, Ottawa, Les Presses de l'Université d'Ottawa, 305-340. 
COLLOQUE INTERNATIONAL SUR LE DÉVELOPPEMENT ÉCONOMIQUE COMMUNAUTAIRE (1992). Compte rendu, Toronto, La Direction du Développement communautaire du ministère des Affaires municipales.

DEFOUNY, J. et J. L. MONZOU CAMPOS (1992). Économie sociale, entre économie capitaliste et économie publique. The Third Sector: Cooperative, Mutual and Nonprofit Organizations, CIRIEC, De Boeck University, Belgique.

DENNIE, D. (1972). Évaluation du programme d'animation socio-culturelle à Sudbury, Ottawa, Secrétariat d'État.

DENNIE, D (1997). Projet de recherche-action, Conseil régional de Sudbury, Conseil de la coopération de l'Ontario, Rapport Final, Département de Sociologie, Université Laurentienne.

FARMER, D. (1996). «Le Centre culturel, acteur privilégié d'une francité renouvelée», B. Cazabon (s. la dir. de ) Pour un espace de recherche au Canada Français, Les Presses de l'Université d'Ottawa, 202-227.

FAVREAU, L. (1989). Mouvement populaire et intervention communautaire de 1960 à nos jours. Continuités et ruptures, Montréal, Alternatives.

FAVREAU, L. et B. LÉVESQUE (1996). Développement économique communautaire, économie sociale et intervention, Presses de l'Université du Québec, Sainte-Foy.

FONTAN, J. M. (1994). «Le développement économique communautaire québécois : éléments de synthèse et point de vue critique», Revue internationale d'action communautaire, vol. 32, no 72,115126.

GARCEAU, M. L., D. DENNIE,V-M. TREMBLAY et M. CHARRON (1991). "Cessons de croire que l'amour va tout vaincre». La situation des femmes francophones de 45 à 64 ans qui vivent en Ontario, La Fédération des femmes canadiennes-françaises de l'Ontario.

GUEST, D. (1995). Histoire de la sécurité sociale au Canada, Beauceville, Éditions Boréal.

JAENEN, Cornelius J. (s. la dir. de) (1993). Les Franco-Ontariens, Ottawa, Les Presses de l'Université d'Ottawa.

JUTEAU, D. et SÉGUIN-KIMPTON, L. (1993). «La collectivité franco-ontarienne : structuration d'un espace symbolique et politique», Cornellius J. Jaenen (s. la dir. de) Les Franco-Ontariens, Ottawa, Les Presses de l'Université d'Ottawa, 265-304.

LAVILLE, J.-L. (dir.). Les services de proximité en Europe, Syros/Alternatives, Paris.

ROSANVALLON, P. (1981). La crise de l'État-providence, Paris, Le Seuil.

SAVARD, R. BEAUCHAMP et P.THOMPSON (1977). Cultiver sa différence Rapport sur les arts dans la vie francophone-ontarienne, Toronto, Conseil des Arts de l'Ontario.

VAILLANCOURT, Y. (1993). «Trois thèses concernant le renouvellement des pratiques sociales dans le secteur public», Nouvelles pratiques sociales, vol. 6, no 1,1-14.

VIENNY, C. (1994). L'économie sociale, Repères, no 148, Éd. La Découverte, Paris.

WELCH,D. (1996). «Survivre en se transformant: les pratiques sociales en Ontario français au cours des années 60 et 70», Nouvelles Pratiques sociales, vol. 9, no 1,151-167.

WOODS-GORDON,A.C.C.O. (1985). Plan d'action, Rapport d'évaluation présenté à l'Assemblée des centres culturels de l'Ontario, Toronto. 


\section{Notes}

1. On pourra consulter Cornelius J. Jaenens (sous la direction de), Les Franco-Ontariens, Ottawa, Les Presses de l'Université d'Ottawa, 1993. Ce livre constitue la première tentative de reconstruire l'histoire, surtout événementielle et institutionnelle, des Franco-Ontariens et Franco-Ontariennes depuis l'arrivée des Français en sol ontarien jusqu'aux années 1990.

2. Nous utilisons la notion de "génération» dans le sens que lui donnent Favreau et Lévesque (1996), à savoir le moment d'apparition, dans une période donnée, de nouvelles organisations, sans pour autant que cela entraîne la disparition des précédentes.

3. Entre 1969 et 1975, les associations culturelles ontaroises se multiplient à un rythme soutenu: une quarantaine de clubs sociaux regroupés en fédération, des dizaines de troupes de théâtres professionnelles et communautaires, plus de quatrevingt centres culturels de tous genres, plusieurs comités culturels, une dizaine de ciné-clubs, etc.

4. En 1969, le gouvernement libéral dirigé par Pierre-Elliott Trudeau adopte la Loi sur les langues officielles du Canada qui accordait aux minorités francophones des droits significatifs visant la protection de leur culture.

5. Certes ce phénomène n'est pas nouveau. Dès le début des années 1980, la question du financement préoccupait sérieusement bon nombre de responsables d'associations communautaires tout comme elle s'était posée pour les écoles privées de langue française dans les années 1960. C'est particulièrement le cas des associations culturelles qui s'étaient réunies dans l'Association des centres culturels de l'Ontario. Fortement appuyées par l'État fédéral à travers des programmes de subventions du Secrétariat d'État destinés aux communautés de langue officielle et par le Bureau franco-ontarien du Conseil des arts de l'Ontario, les associations culturelles, quatre fois plus nombreuses qu'en 1970, voient leur enveloppe budgétaire baisser de façon dramatique au cours des années 1980: le financement public est passé de $52 \%$ du total des recettes en 
1983-1984 à 37 \% des recettes en 19851986 (Farmer, 1996). Les rapports de l'époque (Woods-Gordon, 1985) décrivent le secteur en crise: le peu de ressources dont il dispose sont consacrées à l'administration quotidienne plutôt qu'à la planification des activités, les animateurs se font remplacer graduellement par des gestionnaires de la culture, etc.

6. L'exemple des associations culturelles est un indice révélateur de ces changements. Dès le milieu des années 1980, les acteurs de ce secteur introduisent une perspective entrepreneuriale de la culture qui prône la commercialisation des arts francoontariens, la rationalisation, la transformation des organismes culturels en entreprises. Les organismes culturels comme lieu d'affirmation identitaire deviennent ainsi un simple lieu de consommation. Les associations culturelles, par le biais de l'ACCO (Assemblée des centres culturels), se lancent dans le lobbying auprès des trois paliers du gouvernement pour se faire entendre (Farmer, 1996).

7. On pourra consulter Colloque international sur le Développement économique communautaire. Compte-rendu, Toronto, La Direction du développement communautaire du ministère des Affaires municipales, 1992. 